to have more or less of an impact based on the phrase being searched for.

\section{PTU-145 LOW FODMAP DIET FOR FUNCTIONAL BOWEL DISORDERS. AUDIT OF A NEW SERVICE}

Mimosa Wright*, Rebecca Taylor, Christian Dipper, John Leeds. Newcastle Teaching Hospitals NHS Foundation Trust, Newcastle Upon Tyne, UK

\subsection{6/gutjnl-2018-BSGAbstracts.523}

Introduction Functional bowel disorders are a common reason for referral to gastrointestinal services. A low FODMAP diet has been advocated as an adjunct to management for irritable bowel syndrome (IBS) especially when bloating and/or flatus are a major feature. In the last 12 months in our centre a new dietetic service was started dedicated to providing a low FODMAP diet and this audit was carried out to evaluate the service.

Methods All referrals to the dietitians are recorded on a Trust database and retrospective analysis of these referrals was performed. Patient demographics, source of referral, attendance at the clinic, dietary advice given, symptom response and reason for lack of response were recorded. Univariate analysis was performed to identify factors associated with poor attendance as well as lack of response.

Results 256 patients (mean age 41.5 years, 204 females) were referred to the service of which 188 (73.4\%) attended the clinic. 230/256 (89.8\%) of referrals were from gastroenterology clinics and 26 from other sources (mainly colorectal surgery). Non-attendance at clinic was not associated with sex (13/39 (25.0\%) males vs. 46/195 (23.6\%) females, $\mathrm{p}=0.86)$ or mean age (attenders 41.9 years vs. non-attenders 40.1 years, $\mathrm{p}=0.32)$. There was a trend to non-attendance in those not referred from gastroenterology (10/25 (40.0\%) non-GI patients vs. 49/211 (23.2\%) GI patients, $\mathrm{p}=0.08) .134$ patients had been given dietary advice of which 83 (61.9\%) was a low FODMAP diet and $51(38.1 \%)$ was general IBS advice. To date, 69 patients have completed follow of which 55/69 (79.7\%) reported overall symptomatic improvement. There was no significant difference in improvement rates between the 2 dietary regimes (38/48 FODMAP (79.2\%) vs. 17/21 IBS (80.9\%), $\mathrm{p}=1.0)$. Improvement was not associated with sex (13/17 males vs. $42 / 52$ females, $p=0.73)$ or source of referral ( $2 / 4$ Non-GI vs $53 / 65$ GI, $p=0.18)$. There was a trend to non-improvement in younger patients (improved 44.7 years vs. not improved 37.9 years, $\mathrm{p}=0.08)$. 18 patients described barriers to the diet with $8 / 18$ (44.4\%) not following instructions, 5/18 (27.8\%) describing low mood and 5/18 (27/8\%) describing poor motivation.

Conclusion Dietary advice in functional gut disorders can lead to significant symptom improvement with well selected patients and this new service has been crucial in delivering this. There are few predictors of outcome based on basic clinical features but age may play a role. Further research looking at the interplay of other factors such as quality of life would be useful.

\section{PTU-146 BREATH TESTING FOR GI CANCERS- SCALING UP FOR CLINICAL PRACTICE}

Georgia Woodfield*, Ilaria Belluomo, GengPing Lin, Piers Boshier, Andrea Romano, Wendy Atkin, George B Hanna. Imperial College London, London, UK

\subsection{6/gutjnl-2018-BSGAbstracts.524}

Introduction Gastrointestinal (GI) cancers are a major cause of morbidity and mortality, yet symptoms are common and nonspecific. A non-invasive breath test may be a useful tool for triaging for endoscopy/CT those without red-flag symptoms, or possibly for screening. Prior studies have shown promising results of a breath test for oesophagogastric (80\% sensitivity/ $81 \%$ specificity) and colorectal (96\% sensitivity/76\% specificity) cancers.

The Breath MAGIC (Models for Assessment of GI Cancer) study investigates feasibility and acceptability of breath testing in Primary Care. We also developed a quality control (QC) system for breath sampling.

Methods This is an prospective cohort study of patients attending their GP for current/recent GI symptoms, from November 2016-May 2017, recruitment target 500. Exhaled breath $(250 \mathrm{mls})$ was collected by GP practice/NIHR research nurses using the ReCIVA breath sampling device, onto thermal desorption tubes. Breath volatile organic compounds (VOCs) were analysed using Gas Chromatography (GC) and Proton Transfer Reaction (PTR) Mass Spectrometry, at St Mary's Hospital VOC laboratory. This platform allows analysis of 100 samples per day of continuous, unattended operation. Patients were recruited from 16 London GP practices on the day or were prebooked via phone/text. Feasibility and acceptability were measured using field notes, a telephone conference then focus group of research nurses (thematicially analysed), and GP questionnaires. To develop a QC system, 76 'good quality' samples taken by one experienced operator were used as a standard.

Results Plan-Do-Study-Act cycles from field notes identified barriers and drove regular improvements e.g. phone/text recruitment, a GP poster, grouping of nearby practices, and training healthcare assistants to breath-test. In total 636 patients were enrolled, suggesting breath testing is feasible in Primary Care. Sampling was feasible, with some equipmentrelated but few patient-related limiting factors reported. Analysis was also feasible, with $34 \%$ and $55 \%$ of samples analysed within $<48$ and 72 hours respectively. Two abundant breath compounds, almost universally present, were accurate predictors of adequate sample quality, using acetone $>50 \mathrm{ppb}(\mathrm{H} 3 \mathrm{O}$ +ionisation, PTR) and isoprene $>2.5 \mathrm{ppb}$ ( $\mathrm{NO}$ +ionisation, PTR). This was validated on 284 separate patients' samples. A process was also developed to interrogate ReCIVA software sampling data.

Conclusions Breath testing is feasible in Primary Care, from a human factors and process perspective. This finding, and the development of a QC process, opens up the possibility of large-scale breath testing, pending results of diagnostic accuracy studies. A revised recruitment target of 1000 with new patient acceptability questionniares will likely provide further evidence for this. 